\title{
Catalan's Equation Has No New Solution with Either Exponent Less Than 10651
}

Maurice Mignotte and Yves Roy

\section{CONTENTS}

1. Introduction and Overview

2. Bounding One Exponent as a Function of the Other

3. An Application of Inkeri's First Criterion

Acknowledgement

References
We consider Catalan's equation $x^{p}-y^{q}=1$ (where all variables are integers and $p, q$ are greater than 1 ), which has the obvious solution $9-8=1$. Are there others? Applying old and new theoretical results to a systematic computation, we were able to improve on recent work of Mignotte and show that Catalan's equation has only the obvious solutions when $\min \{p, q\}<10651$. Two crucial tools used are a new result of Laurent, Mignotte, and Nesterenko on linear forms of logarithms, and a criterion obtained by W. Schwarz in 1994.

\section{INTRODUCTION AND OVERVIEW}

In 1843, Eugène Catalan considered the following question: Are there pairs of consecutive integers that are both powers, other than $(-1,0),(0,1)$ and $(8,9)$ ? The general opinion, known as Catalan's conjecture, is that the answer is no. Formally, the relevant diophantine equation is $x^{m}-y^{n}=1$, with $x, y$ are integers and $m, n$ integers greater than 1 . Of course, we can assume that the exponents are prime numbers, and, possibly after interchanging the two terms on the left, that $x$ and $y$ are both nonnegative. Excluding the trivial case of $x=1$ and $y=0$, the equation we are interested in is

$$
x^{p}-y^{q}=1,
$$

where $p, q$ are prime numbers and $x, y$ are integers greater than 1 .

The main results toward the verification of Catalan's conjecture are of relatively recent vintage (see [Ribenboim 1994] for a more detailed account). An important step was taken by Tijdeman [1976], who proved that the problem is finite: using Baker's results on linear forms in logarithms, he showed that 
all unknowns are effectively bounded. The same year, Langevin [1977] obtained the explicit bound $10^{110}$ for $\max \{p, q\}$, and enormous bounds for $x$ and $y$. Since then progress on linear forms has led to better bounds. Two years ago, it was possible to prove $\max \{p, q\}<10^{21}$, and now it seems that $\max \{p, q\}<10^{18}$ could be proved. However, we shall not pursue upper bounds in the present paper, but will focus our attention on improving the known lower bounds on $\min \{p, q\}$.

The first result in this direction [Nagell 1920] was $\min \{p, q\} \neq 3$. Almost half a century elapsed until Ko Chao proved that $\min \{p, q\} \neq 2[$ Ko 1965]. The best result published since then [Mignotte 1994] had been

$$
\min \{p, q\} \geq 13 \text {. }
$$

Here we report a significant advance, proving that

$$
\min \{p, q\} \geq 10651 \text {. }
$$

This result was obtained thanks to several theoretical advances and a lot of computation. To explain our strategy, it is convenient to generalize (1.1) slightly to

$$
x^{p}-y^{q}=\varepsilon, \text { with } \varepsilon= \pm 1 \text { and } x, y>1
$$

(still assuming $p, q$ prime). This is so that we can interchange the roles of $(x, p)$ and $(y, q)$ as needed.

The first theoretical advance, discussed in Section 2 , is a new lower bound for two linear forms in logarithms [Laurent et al.]. Applied to (1.2) for a fixed prime $p$, it leads to an upper bound

$$
q<q_{\max }(p) .
$$

We have made great efforts to get a good value for this bound, in order to decrease computation time for the present work and to help the future improvement of upper bounds on $\max \{p, q\}$. In the process (Section 2.1) we present a technical refinement of the congruences obtained in 1964 by Hyyrö.

Then, for a fixed $p$, we have to consider the range $q<q_{\max }(p)$. For each pair $(p, q)$ we have several theoretical tools to attack (1.2), which in most cases are sufficient to eliminate the possibility of solutions. Specifically, for each prime $p$ one can define two sets $F(p)$ and $H(p)$ such that a solution of (1.2) can only exist for

$$
q \in F(p) \cup H(p) .
$$

The first set corresponds to Fermat quotients:

$$
F(p)=\left\{q: p^{q-1} \equiv 1 \bmod q^{2}\right\} .
$$

Experiments show that this set is generally very small, but its computation takes a very long time. In our case, to compute all these sets for $p<10625$ took more than two weeks on a parallel computer with 32 processors. The reason for the strange value 10625 is purely technical: the program was written in $\mathrm{C}$, in double precision, and 10625 is the highest value for which we can compute congruences $\bmod q^{2}$ with this program.

The second set $H(p)$ is related to certain class numbers, and comes from the first general algebraic criterion on Catalan's equation, obtained by Inkeri [1990]. Inkeri's criterion allows us to put

$$
H(p)=\left\{q<q_{\max }(p): q \text { divides } h\left(K_{p}^{\prime}\right)\right\},
$$

where $h(K)$ represents the class number of a number field $K$ and

$$
K_{p}^{\prime}= \begin{cases}\mathbb{Q}[\sqrt{-p}] & \text { if } p \equiv 3 \bmod 4 \\ \mathbb{Q}\left[e^{2 i \pi / p}\right] & \text { if } p \equiv 1 \bmod 4\end{cases}
$$

The case $p \equiv 1 \bmod 4$ leads to very serious difficulties; the class number of $\mathbb{Q}\left[e^{2 i \pi / p}\right]$ is not known for $p \geq 71$. There is a way to overcome this problem: Given $q$, and setting $h_{p}=h\left(\mathbb{Q}\left[e^{2 i \pi / p}\right]\right)$, there are procedures that output either the answer " $q$ does not divide $h_{p}$ " or " $q$ may divide $h_{p}$ ". But these procedures are very slow. In April 1993, Mignotte [1995] was able to replace the field $K_{p}^{\prime}$ in the previous criterion by

$$
K_{p}=\text { the subfield of } \mathbb{Q}\left[e^{2 i \pi / p}\right] \text { of degree } 2^{d},
$$

where $2^{d}$ is the maximal power of 2 in $p-1$. For many values of $p$ the degree of this new field $K_{p}$ is much smaller than $p-1$, and $h\left(K_{p}\right)$ can be easily 
computed. But there are still difficult examples, like $p=257$, where this degree is 256 .

The newest result we use is from [Schwarz 1995], to the effect that in $(1.3)$ we can replace $h\left(K_{p}\right)$ by $h^{-}\left(K_{p}\right)$, the relative class number of $K_{p}$ over $K_{p}^{+}$(that is, the quotient $h\left(K_{p}\right) / h\left(K_{p}^{+}\right)$; here $K_{p}^{+}$ is the maximal real subfield of $\left.K_{p}\right)$. This represents an enormous progress from the computational point of view: one can compute $h^{-}\left(K_{p}\right)$ for any $p$ [Washington 1982]. Without this improvement we had serious computational difficulties to get $\min \{p, q\}>570$, whereas now the most expensive computational step is computing the Fermat quotients.

To summarize the discussion so far, we eliminate most possibilities for $(p, q)$ by using the bound $q<$ $q_{\max }(p)$ and the following fact:

Criterion 1.1. Let $p$ and $q$ be odd prime numbers. Let $p-1=2^{d} l$, where $l$ is odd. Let $K=K_{p}$ be the subfield of $\mathbb{Q}\left[e^{2 i \pi / p}\right]$ of degree $2^{d}$. Denote by $h_{K}^{-}$the relative class number of $K$ over $K^{+}$. Then (1.2) has no solution when both of these relations are satisfied:

$$
q \nmid h_{K}^{-} \quad \text { and } \quad p^{q-1} \not \equiv 1 \bmod q^{2} .
$$

Now suppose that, for a given $p$, we want to analyze a value of $q$ that does not satisfy Criterion 1.1 (that is, $q \in F(p) \cup H(p)$ ). We have two ways of attack. The more natural, and generally quicker, way is to try Criterion 1.1 on the pair $(q, p)$. We illustrate with the first values of $p$. For $p=5$, we have $q_{\max }(5)=110000, F(5)=\{20771,40487\}$, and $H(5)=\varnothing$; we therefore consider $p=20771$ and $p=40487$, and examine the possibility of $q=5$. Since

$$
\begin{array}{ll}
5 \notin F(20771), & 5 \notin H(20771)=\{41\}, \\
5 \notin F(40487), & 5 \notin H(40487)=\{179\},
\end{array}
$$

we conclude that (1.2) has no solution when $p=5$. Similarly, for $p=7$, we have $q_{\max }(7)=110000$, $F(7)=\{5\}$, and $H(7)=\varnothing$; since we already know that $p=5$ leads to no solution, we conclude that $p=7$ also leads to no solution.
Sometimes this strategy fails; the smallest example, already noticed by Inkeri [1964], is the pair $(p, q)=(83,4871)$, because

$$
4871 \in F(83) \quad \text { and } \quad 83 \in F(4871) .
$$

In such cases, we try to use the following elementary criterion from [Mignotte 1993]:

Criterion 1.2. Let $p$ and $q$ be odd prime numbers, and let $l$ be a prime number such that $l=h p q+1$, with $h$ a positive integer. Let $a$ and $b$ be integers such that $a p \equiv 1 \bmod l$ and $b q \equiv 1 \bmod l$. Then (1.2) has no solution when all the following relations are satisfied: $q^{h q} \not \equiv 1 \bmod l, p^{h p} \not \equiv 1 \bmod l$, and

$$
\left(\left(1+a g^{j q}\right)^{p}-1\right)^{h p} \not \equiv 1 \bmod l
$$

for all $j \in\{0,1, \ldots, h p-1\}$, where $g$ is a primitive root $\bmod l$.

For all pairs $(p, q)$ unresolved by the use of Criterion 1.1 (with $p<10651$ ), the use of Criterion 1.2 was sufficient to show the absence of solutions, except for the pair $(2903,18787)$. This last case could be solved by congruences mod 327231967 applied to the formulas obtained during the proof of the first criterion of Inkeri; the details are too technical to be given here.

The conclusion of our computations is, therefore:

Theorem 1.3. Catalan's equation

$$
x^{p}-y^{q}=1,
$$

where $p$ and $q$ are primes and $x, y>1$ are integers, has no solutions other than $9-8=1$ when $\min \{p, q\}<10651$.

The computed data can be obtained from the authors.

In Section 2 we derive the bound $q<q_{\max }(p)$ that makes the problem tractable. In Section 3 we present a result that is not used in the proof of Theorem 1.3, but shows that the special case of Catalan's equation with exponents congruent to 3 mod 4 could be simpler than the general case. 


\section{BOUNDING ONE EXPONENT AS A FUNCTION OF THE OTHER}

\section{Arithmetical Relations}

Suppose $(x, y, p, q)$ is a solution to Catalan's equation (1.2). Cassels [1960] proved that there exist integers $r$ and $s$ such that

$$
y+\varepsilon=\frac{s^{p}}{q} \quad \text { and } \quad x-\varepsilon=\frac{r^{q}}{p} .
$$

According to [Hyyrö 1964], there exist also integers $a_{0} \geq 1$ and $u_{0} \geq 2$ such that $a=q a_{0}-\varepsilon$ and $u=p^{q-1} u_{0}+1$ satisfy

$$
x-\varepsilon=p^{q-1} a^{q} \quad \text { and } \quad x^{p}-\varepsilon=(p u a)^{q} .
$$

(Hyyrö gives additional relations satisfied by these numbers, but we will not need them.)

Since $u>2 p^{q-1}$, we get

$$
x^{p}-\varepsilon>\left(2 p^{q} a\right)^{q} \geq\left(2 p^{q}(q-1)\right)^{q},
$$

so that

$$
x^{p} \geq\left(2(q-1) p^{q}\right)^{q} .
$$

This implies

$$
r^{q}=p(x-\varepsilon)>x-1 \geq\left((q-1) p^{q}\right)^{q / p} \geq p^{q^{2} / p},
$$

whence

$$
\log r>\frac{q}{p} \log p
$$

This lower bound seems to be new. In any case, it is quite useful for the estimates in the remainder of this section.

\section{A Crude Bound}

It is easy to prove that $s \leq 4^{1 / p} q^{1 / q} r$ and $r \leq$ $4^{1 / p} p^{1 / p} s$, and also that the linear form

$$
\Lambda=p \log p-q \log \frac{q^{p}}{s^{p}-q \varepsilon}
$$

satisfies $0<|\Lambda| \leq 4 p^{2} r^{-q}$. Let's assume that

$$
q>\max \{400 p \log p, 90000 \log p\} .
$$

Combined with (2.2), this implies

$$
\log |\Lambda| \leq-0.999999 q \log r .
$$

We shall apply the following result from [Laurent et al.], where, for $\alpha$ an algebraic number, $h(\alpha)=$ $\log M(\alpha) / \operatorname{deg} \alpha$ is the logarithmic height of $\alpha$ (here $M(\alpha)$ is the Mahler measure of $\alpha$, the definition of which is recalled on page 267 ).

Theorem 2.1. Let $\alpha_{1}, \alpha_{2}$ be two multiplicatively independent algebraic numbers with $\left|\alpha_{1}\right|,\left|\alpha_{2}\right| \geq 1$, and let $\log \alpha_{1}$ and $\log \alpha_{2}$ be any determination of their logs. Put

$$
\Lambda=b_{2} \log \alpha_{2}-b_{1} \log \alpha_{1},
$$

where $b_{1}$ and $b_{2}$ are positive integers. Put

$$
D=\frac{\left[\mathbb{Q}\left(\alpha_{1}, \alpha_{2}\right): \mathbb{Q}\right]}{\left[\mathbb{R}\left(\alpha_{1}, \alpha_{2}\right): \mathbb{R}\right]} .
$$

Let $K$ be an integer $\geq 2$, let $L, R_{1}, R_{2}, S_{1}, S_{2}$ be positive integers, and let $\rho>1$ be a real number. Suppose that

$$
R_{1} S_{1} \geq L \quad \text { and } \quad R_{2} S_{2}>(K-1) L .
$$

Put $R=R_{1}+R_{2}-1, S=S_{1}+S_{2}-1$,

$$
g=\frac{1}{4}-\frac{K L}{12 R S}
$$

and

$$
b=\left((R-1) b_{2}+(S-1) b_{1}\right)\left(\prod_{k=1}^{K-1} k !\right)^{-2 /\left(K^{2}-K\right)} .
$$

Suppose also that

$$
(\rho-1) \log \alpha_{i}+2 D h\left(\alpha_{i}\right) \leq a_{i} \quad \text { for } i=1,2,
$$

that the numbers $r b_{2}+s b_{1}$, for $0 \leq r \leq R-1$ and $0 \leq s \leq S-1$, are pairwise distinct, and that

$K(L-1) \log \rho-(D+1) \log K L$

$$
-D(K-1) \log (b / 2)-g L\left(R a_{1}+S a_{2}\right)>0 \text {. }
$$

Then we have the lower bound

$$
\left|\Lambda^{\prime}\right| \geq \rho^{-K L+\frac{1}{2}}
$$

where

$$
\Lambda^{\prime}=\Lambda \max \left\{\frac{L S e^{L S|\Lambda| /\left(2 b_{2}\right)}}{2 b_{2}}, \frac{L R e^{L R|\Lambda| /\left(2 b_{1}\right)}}{2 b_{1}}\right\}
$$


Before applying Theorem 2.1, we apply a corollary of it [Laurent et al., Corollary 2], which is weaker but much simpler to use.

Corollary 2.2. With the notations of Theorem 2.1, suppose moreover that $\alpha_{1}$ and $\alpha_{2}$ are positive real numbers. Then

$\log |\Lambda| \geq-24.34 D^{4}\left(\max \left\{\log b^{\prime}+0.5, \frac{21}{D}\right\}\right)^{2} \log A_{1} \log A_{2}$, where

$\log A_{i} \geq \max \left\{\frac{1}{D}, \frac{\left|\log \alpha_{i}\right|}{D}, h\left(\alpha_{i}\right)\right\} \quad$ for $i=1,2$, and $b^{\prime}=\frac{b_{1}}{\log A_{2}}+\frac{b_{2}}{\log A_{1}}$.

We apply the corollary with $b_{1}=q, b_{2}=p$,

$$
\alpha_{1}=\frac{q r^{p}}{\left(s^{p}-\varepsilon q\right)}
$$

$\alpha_{2}=p$, and $D=1$. Notice that $\alpha_{1}$ and $\alpha_{2}$ are multiplicatively independent: otherwise $\Lambda$ would be an integer times $\log p$, contradicting the trivial estimate $0<|\Lambda|<1$.

Notice also that

$$
\left|\log \alpha_{1}\right| \leq \frac{p \log p}{q}+\frac{|\Lambda|}{q} \leq \frac{p}{q} \log (p+1) .
$$

Moreover,

$$
\begin{aligned}
h\left(\alpha_{1}\right) & \leq \max \left\{p \log r+\log q, \log \left(s^{p}+q\right)\right\} \\
& \leq \max \left\{(p+1) \log r, p \log s+2^{-p}\right\} \\
& \leq(p+1) \log r,
\end{aligned}
$$

since $q \leq s$. Clearly, $\log \alpha_{2}=h\left(\alpha_{2}\right)=\log p$. Thus we can apply Corollary 2.2 with

$$
\log A_{1}=(p+1) \log r \quad \text { and } \quad \log A_{2}=\log p .
$$

(Note that to apply Corollary 2.2 we only have to choose $\log A_{1}$ and $\log A_{2}$. Then $b^{\prime}$ is defined in terms of $b_{1}, b_{2}, \log A_{1}$ and $\log A_{2}$. The corollary gives a lower bound for $\Lambda$ depending only on these previous quantities and on $D$.)

Hence, by (2.3), we have

$$
b^{\prime}=\frac{q}{\log p}+\frac{p}{(p+1) \log r} \leq \frac{1.001 q}{\log p} .
$$

We get

$$
\begin{array}{r}
\log |\Lambda| \geq-24.34(\max \{21, \log (q / \log p)+0.51\})^{2} \\
\times(p+1) \log p \log r .
\end{array}
$$

Comparing this inequality with (2.4) leads to

$q \leq 24.4(p+1) \log p(\max \{21, \log (q / \log p)+0.51\})^{2}$.

In particular, $q \leq 170000$ for $p \leq 7$.

\section{A Sharper Bound}

In this section we assume $p \geq 11$. We can apply Theorem 2.1 with

$$
a_{1}=2(p+1)\left(1+\frac{(\rho-1)}{4 q}\right) \log r
$$

and $a_{2}=(\rho+1) \log p$. We shall take $17 \leq \rho \leq 25$. By (2.2) and (2.3), we have $a_{1}>2 q \log p$, so that $a_{1}>1.03 \times 10^{5}, a_{2}>43.16$, and $a_{1} a_{2}>3.51 \times 10^{7}$. Then, to satisfy condition (2.5), we take

$$
\begin{aligned}
& R_{1}=1, \quad S_{1}=L, \\
& R_{2}=\left[\sqrt{(K-1) L a_{2} / a_{1}}\right]+1, \\
& S_{2}=\left[\sqrt{(K-1) L a_{1} / a_{2}}\right]+1 .
\end{aligned}
$$

We suppose that $7 \leq L \leq 5 \log p$. We take $K=$ $\left[\mu^{2} L a_{1} a_{2}\right]+1$, where $\mu$ is some real number to be chosen later, satisfying $0.2 \leq \mu \leq 0.5$; thus

$$
K \geq 0.2^{2} \times 7 \times 1.03 \times 10^{6} \times 43.16>1.24 \times 10^{7} .
$$

If there exist two integers $r_{0}$ and $s_{0}$, with $\left|r_{0}\right|<$ $R$ and $\left|s_{0}\right|<S$, such that $r_{0} b_{2}+s_{0} b_{1}=0$, then $q$ divides $r_{0}$, so that

$$
\begin{aligned}
q & <R \leq 1.5 \mu L(\rho+1) p \\
& <1.5 \times 0.5 \times 5 \times 26 \times p \log p<100 p \log p,
\end{aligned}
$$

which contradicts (2.3). Hence, the numbers

$$
r b_{2}+s b_{1}
$$

for $0 \leq r \leq R-1$ and $0 \leq s \leq S-1$, are pairwise distinct.

We have the following general upper bound for $b$ [Laurent et al., Lemma 6]: 


$$
\begin{aligned}
b \leq & \frac{\left((R-1) b_{2}+(S-1) b_{1}\right)}{K-1} \\
& \times \exp \left(\frac{3}{2}-\frac{\log (2 \pi(K-1) / \sqrt{e})}{K-1}+\frac{\log K}{6 K(K-1)}\right) .
\end{aligned}
$$

Thanks to our hypotheses on $L$ and $K$, this leads to

$$
\begin{array}{r}
\log b \leq 1.5+\log \left(\sqrt{L}(\sqrt{K}+1)\left(b_{1} \sqrt{a_{1} / a_{2}}+b_{2} \sqrt{a_{2} / a_{1}}\right)\right) \\
-\log (K-1)-\frac{\log (3.8 K)}{K-1} \\
\leq 1.5+\log \left(\frac{\sqrt{L} \sqrt{a_{1} a_{2}}}{(\rho+1)(\sqrt{K}-1)}\right)+\log b^{\prime}-\frac{\log (3.8 K)}{K-1} \\
\leq 1.5-\log ((\rho+1) \mu)+\log b^{\prime}+\frac{1}{\sqrt{K-1}}-\frac{\log (3.8 K)}{K-1} \\
\leq 1.5-\log ((\rho+1) \mu)+\log \frac{q}{\log p} \\
+\frac{(\rho+1) p}{2 q^{2}}+\frac{1}{\sqrt{K-1}}-\frac{\log (3.8 K)}{K-1},
\end{array}
$$

since now

$$
\begin{aligned}
b^{\prime} & =(\rho+1)\left(\frac{b_{1}}{a_{2}}+\frac{b_{2}}{a_{1}}\right) \\
& \leq \frac{q}{\log p}+\frac{p(\rho+1)}{2(p+1) \log r} \leq \frac{q}{\log p}\left(1+\frac{(\rho+1) p}{2 q^{2}}\right) .
\end{aligned}
$$

Now we consider the quantity $g$ of (2.6). From the relations

$$
\begin{aligned}
& R=R_{1}+R_{2}-1 \leq \sqrt{(K-1) L a_{2} / a_{1}}, \\
& S=S_{1}+S_{2}-1 \leq L+\sqrt{(K-1) L a_{1} / a_{2}},
\end{aligned}
$$

we get

$$
\begin{aligned}
& g L\left(R a_{1}+S a_{2}\right) \\
& \quad=\frac{1}{4} L\left(R a_{1}+S a_{2}\right)-\frac{K L^{2}}{12}\left(\frac{a_{1}}{S}+\frac{a_{2}}{R}\right) \\
& \leq \frac{1}{4} L^{2} a_{2}+\frac{1}{2} L^{3 / 2} \sqrt{(K-1) a_{1} a_{2}}-\frac{K L^{2}}{12}\left(\frac{a_{1}}{S}+\frac{a_{2}}{R}\right) .
\end{aligned}
$$

We have

$$
\frac{1}{R} \geq \frac{1}{\sqrt{(K-1) L a_{2} / a_{1}}}
$$

and the identity

$$
\frac{1}{x+y}=\frac{1}{x}-\frac{y}{x^{2}}+\frac{y^{2}}{(x+y) x^{2}}
$$

implies

$$
\begin{aligned}
\frac{1}{S} \geq \frac{1}{\sqrt{(K-1) L a_{1} / a_{2}}}-\frac{L}{(K-1) L a_{1} / a_{2}} \\
+\frac{a_{2} L^{2}}{a_{1}(K-1) L\left(L+\sqrt{(K-1) L a_{1} / a_{2}}\right)}
\end{aligned}
$$

Hence we obtain the lower bound

$$
\begin{aligned}
& K L^{2}\left(\frac{a_{1}}{S}+\frac{a_{2}}{R}\right) \geq(K-1) L^{2}\left(\frac{a_{1}}{S}+\frac{a_{2}}{R}\right) \\
& \geq 2 L^{3 / 2} \sqrt{(K-1) a_{1} a_{2}}-a_{2} L^{2} \\
&+\frac{a_{2} L^{3}}{L+\sqrt{(K-1) L a_{1} / a_{2}}} .
\end{aligned}
$$

Plugging this into (2.10) gives

$$
\begin{aligned}
g L\left(R a_{1}+S a_{2}\right) \leq \frac{1}{3} L^{3 / 2} \sqrt{(K-1) a_{1} a_{2}} \\
\quad+\frac{1}{3} L^{2} a_{2}-\frac{a_{2} L^{3}}{12\left(L+\sqrt{(K-1) L a_{1} / a_{2}}\right)} .
\end{aligned}
$$

Ignoring the last term, we get

$$
g L\left(R a_{1}+S a_{2}\right) \leq \frac{1}{3} L^{3 / 2} \sqrt{(K-1) a_{1} a_{2}}+\frac{1}{3} a_{2} L^{2} .
$$

Besides, since $\log r>(q / p) \log p$, we have

$$
\frac{a_{2}}{a_{1}} \leq \frac{(\rho+1) \log p}{2(p+1) \log r}<\frac{(\rho+1) \log p}{2(p+1)(q / p) \log p}<\frac{\rho+1}{2 q} .
$$

Using these remarks, we see that condition (2.7) is satisfied if, putting $\lambda=\log \rho$, we have

$$
\begin{aligned}
0< & K(L-1) \lambda+(K-1) \log 2-2 \log (K L) \\
- & \frac{1}{3} L^{3 / 2} \sqrt{(K-1) a_{1} a_{2}}-\frac{1}{3} a_{2} L^{2} \\
- & (K-1)\left(1.5-\log ((\rho+1) \mu)+\log \left(\frac{q}{\log p}\right)\right. \\
& \left.+\frac{(\rho+1) p}{2 q^{2}}+\frac{1}{\sqrt{K-1}}-\frac{\log (3.8 K)}{K-1}\right) .
\end{aligned}
$$

Now the right-hand side of (2.11) is greater than or equal to $\Phi+\Theta$, where

$$
\begin{aligned}
\Phi= & K(L-1) \lambda+K \log 1.999-\frac{1}{3} L^{3 / 2} \sqrt{(K-1) a_{1} a_{2}} \\
& -(K-1)\left(1.5-\log ((\rho+1) \mu)+\log \left(\frac{q}{\log p}\right)\right)
\end{aligned}
$$


and

$\Theta=5 K \times 10^{-4}-\log \left(K L^{2}\right)-K \frac{(\rho+1) p}{q^{2}}-\sqrt{K}-\frac{1}{3} a_{2} L^{2}$.

It is easy to verify that $\Theta$ is positive. Indeed,

$$
\begin{aligned}
\frac{(\rho+1) p}{q^{2}} & \leq \frac{26}{90000 \times 400 \times \log ^{2} p}<2 \times 10^{-7}, \\
\frac{\sqrt{K}}{K} & \leq 2.84 \times 10^{-4}, \\
\frac{\log \left(K L^{2}\right)}{K} & <3 \frac{\log K}{K}<4 \times 10^{-6}, \\
\frac{\frac{1}{3} a_{2} L^{2}}{K} & \leq \frac{a_{2} L^{2}}{35^{2} L a_{1} a_{2}}=\frac{L}{35^{2} a_{1}} \leq \frac{5 \log p}{6 \times 0.2^{2} \times q \log p} \\
& <\frac{5}{6 \times 0.2^{2} \times 200000}<1.1 \times 10^{-4},
\end{aligned}
$$

so $\Theta \geq K(5-4.6) \times 10^{-4}>0$. Then, dividing $\Phi$ by $L a_{1} a_{2}$, we see that condition (2.7) is satisfied when

$$
\begin{array}{r}
\mu\left((L-1) \lambda+\log 1.999-1.5+\log (\rho+1)-\log \frac{q}{\log p}\right) \\
+\mu \log \mu-\frac{1}{3} L \geq 0 . \quad(2.12)
\end{array}
$$

In such a case, by (2.8) and the inequality $R+S \leq$ $K$, we get

$$
\log |\Lambda| \geq-K L \log \rho-\log (K L) .
$$

Comparing this inequality with (2.4) gives

$$
q \leq 2.0001 \mu^{2} L^{2}(\rho+1)(p+1) \log p \log \rho .
$$

Now we can describe the procedure used to get an upper bound $q_{\max }(p)$ for the exponent $q$ in (1.2), when $p$ is fixed. We first apply condition (2.9) to get a first upper bound, say $q_{0}$, for this exponent. Then, for a suitable choice of $\rho$ and $L$, we use this upper bound to find a value $\mu$ for which (2.11) holds. Then (2.11) gives an upper bound $q_{1} \leq q_{0}$. If $q_{1}<q_{0}$ we repeat this process using the new upper bound $q_{1}$ and some choice of $\rho$ and $L$ (possibly the same as before), which gives an upper bound $q_{2} \leq q_{1}$. We continue in this way, and stop after a certain number of tries, obtaining a value $q_{\infty}$. Finally we take

$$
q_{\max }(p)=\max \left\{90000 \log p, 400 p \log p, q_{\infty}\right\},
$$

in order to respect $(2.3)$. Notice that $q_{\max }(p)=$ $90000 \log p$ for $p \leq 53$.

Since $\lambda=\log p$, condition (2.12) is equivalent to $\mu\left(L \log \rho-\theta+\log \left(1+\frac{1}{\rho}\right)-\log \frac{q}{\log p}\right)+\mu \log \mu-\frac{1}{3} L \geq 0$, where we put $\theta=1.5-\log 1.999$. This can also be written as

$$
L\left(\mu \log \rho-\frac{1}{3}\right) \geq \mu\left(\theta-\log \left(1+\frac{1}{\rho}\right)+\log \frac{q}{\log p}\right) .
$$

We choose

$$
\mu=\frac{2}{3 \log \rho} ;
$$

then the previous condition becomes

$$
L \geq 3 \times \frac{2}{3 \log \rho}\left(\theta-\log \left(1+\frac{1}{\rho}\right)+\log \frac{q}{\log p}\right) .
$$

For $\rho=22.9$ (so that $\mu=0.2129 \ldots \in[0.2,0.5]$ ), we find that this inequality holds if

$$
L \geq 0.6388(\log q-\log \log p+0.765),
$$

and we can take

$$
L=[0.6388 \log (q / \log p)+1.49] .
$$

(We verify the condition $7 \leq L \leq 5 \log p$. From (2.3), we have

$$
L \geq 0.6388 \log 90000>7 .
$$

Put $z=q / \log p$; then (2.3) implies $z>11.407$ and

$$
\max \{21, \log (q / \log p)+0.51\}<1.841 z ;
$$

thus (2.9) gives

$$
z \leq 24.4 \times 1.841^{2}(p+1) \log ^{2} z,
$$

which leads to

$$
\log z<\log 82.7+\log (p+1)+2 \log \log z
$$

and

$$
\log z<1.746 \log (82.7(p+1)) .
$$


Then an elementary numerical study shows that $L<4 \log p$ for $p \geq 11$. This ends the verification.)

Thus we get

$q \leq 6.7853(p+1)(0.6388 \log (q / \log p)+1.49)^{2} \log p$,

which implies

$$
q \leq 2.769(p+1)(\log (q / \log p)+2.333)^{2} \log p
$$

for $p \geq 11$; thus

$$
q \leq 2.77 p(\log (q / \log p)+2.333)^{2} \log p
$$

for $p \geq 3000$.

On the range $11 \leq p<10651$, we have computed the best possible value $q_{\max }(p)$ obtained by Theorem 2.1. Inequality (2.14) is given as a reference for possible further computations. Example: for $p<10^{4}$ we have $q_{\max }(p)<8.7 \times 10^{7}$.

\section{AN APPLICATION OF INKERI'S FIRST CRITERION}

We now prove a result that is not used in the proof of Theorem 1.3, but shows that the special case of Catalan's equation with exponents congruent to 3 mod 4 could be simpler than the general case.

Instead of (1.2) we will work with the equation $x^{p}-y^{q}=1$, where $q>p>1$ are positive integers and $x, y$ are (possibly negative) integers with $|x|$, $|y|>1$. We will in fact assume that $p>50$.

We recall briefly the work in [Inkeri 1964]. For $p$ prime, with $p \equiv 3 \bmod 4$, suppose that $a$ runs over the quadratic residues mod $p$ and that $b$ runs over the nonresidues. Put

$$
A(X)=\prod_{a}\left(X-\zeta^{a}\right), \quad B(X)=\prod_{b}\left(X-\zeta^{b}\right),
$$

where $\zeta=e^{2 i \pi / p}$. Then

$$
4 \frac{X^{p}-1}{X-1}=2 A(X) \cdot 2 B(X)=Y^{2}(X)+p Z^{2}(X),
$$

where

$$
\begin{aligned}
& Y(X)=A(X)+B(X) \\
& Z(X)=(B(X)-A(X)) / \sqrt{-p}
\end{aligned}
$$

The polynomials $Y$ and $Z$ have integer coefficients. Clearly, $\operatorname{deg} Y=\frac{1}{2}(p-1)$ and

$$
\begin{aligned}
Y(X) & =2 X^{(p-1) / 2}+\cdots, \\
L(Y) & \leq L(A)+L(B) \leq 2^{(p+1) / 2},
\end{aligned}
$$

where $L(P)$ denotes the length of the polynomial $P$ (that is, the sum of the modules of its coefficients).

From the formula on Gauss sums,

$$
\sum_{a} \zeta^{a}-\sum_{b} \zeta^{b}=\sqrt{-p}
$$

we see that $\operatorname{deg} Z=\frac{1}{2}(p-3)$ and that

$$
\begin{aligned}
Z(X) & =X^{(p-3) / 2}+\cdots, \\
L(Z) & \leq(L(A)+L(B)) / \sqrt{p} \leq 2^{(p+1) / 2} / \sqrt{p} .
\end{aligned}
$$

Now, by Hyyrö's theorem (see the beginning of Section 2), there exist integers $a$ and $u$ such that

$$
|x|-1=p^{q-1} a^{q} \quad \text { and } \quad|x|^{p}-1=(|x|-1) p u^{q} .
$$

Thus $4 p u^{q}=Y^{2}+p Z^{2}$ and, if $Y_{1}=Y /(2 p)$ and $Z_{1}=\frac{1}{2} Z$, then

$$
u^{q}=\left(Z_{1}+Y_{1} \sqrt{-p}\right)\left(Z_{1}-Y_{1} \sqrt{-p}\right) ;
$$

moreover $Y_{1}$ and $Z_{1}$ are coprime integers [Inkeri 1964]. In the quadratic field $\mathbb{Q}(\sqrt{-p})$, this implies a relation

$$
\left(Z_{1}+Y_{1} \sqrt{-p}\right)=\mathfrak{b}^{q}
$$

where $\mathfrak{b}$ is some ideal of this field. If we assume that $q$ does not divide the class number of $\mathbb{Q}(\sqrt{-p})$, there exists an algebraic integer $\beta$, belonging to this field, such that

$$
Z_{1}+Y_{1} \sqrt{-p}=\beta^{q} .
$$

Hence, $\beta^{q}-\bar{\beta}^{q}=2 Y_{1} \sqrt{-p}$ and $\beta^{q}+\bar{\beta}^{q}=2 Z_{1}$. Put $\beta=|\beta| e^{i \theta}$, with $|\theta| \leq \pi$. Then

$$
\cot (q \theta)=i \frac{\beta^{q}+\bar{\beta}^{q}}{\beta^{q}-\bar{\beta}^{q}}=\frac{Z(|x|) \sqrt{p}}{Y(|x|)} .
$$


Using the previous estimates relative to $Y$ and $Z$, we get

$$
\begin{aligned}
|\cot (q \theta)| & <\sqrt{p} \frac{|x|^{(p-3) / 2}+2^{(p+1) / 2}|x|^{(p-5) / 2}}{2|x|^{(p-1) / 2}-2^{(p+1) / 2}|x|^{(p-3) / 2}} \\
& =\sqrt{p} \frac{1+2^{(p+1) / 2} /|x|}{2|x|\left(1-2^{(p+1) / 2} /|x|\right)}<\frac{2 \sqrt{p}}{3|x|},
\end{aligned}
$$

since $|x|>p^{p}$ by an argument like the one leading to (2.1). Thus there exists an integer $k$ such that the linear form $\Lambda:=k i \pi-q(2 i \theta)$ satisfies

$$
|\Lambda|<\frac{2 \sqrt{p}}{|x|} .
$$

We now use [Laurent et al., Theorem 3]:

Theorem 3.1. Let $\alpha$ be an algebraic number of modulus 1 that is not a root of unity, let $b_{1}$ and $b_{2}$ be two positive integers, and set $\Lambda=b_{1} i \pi-b_{2} \log \alpha$. Put $D=\frac{1}{2}[\mathbb{Q}(\alpha): \mathbb{Q}]$,

$$
\begin{aligned}
t & \geq \max \{20,12.85|\log \alpha|+D h(\alpha)\}, \\
H & =\max \left\{17, D \log \left(\frac{b_{1}}{2 a}+\frac{b_{2}}{25.7 \pi}\right)+4.6 D+3.25\right\} .
\end{aligned}
$$

Then $\log |\Lambda| \geq-9 t H^{2}$.

In our case we take $b_{1}=k, b_{2}=q, \alpha=\beta / \bar{\beta}=e^{2 i \theta}$, and $D=1$.

For an algebraic number $\gamma$, let $M(\gamma)$ denote the Mahler measure of $\gamma$, that is, the product

$$
\left|a_{0}\right| \prod_{j=1}^{d} \max \left\{1,\left|\gamma_{j}\right|\right\},
$$

where $a_{0}$ is the leading coefficient and the $\gamma_{j}$ the roots of an irreducible polynomial with integer coefficients of which $\gamma$ is a root. We have the estimates

$$
\begin{aligned}
M(\alpha) & =|\beta|^{2} \leq\left(\left|Z_{1}\right|+\left|Y_{1}\right| \sqrt{p}\right)^{2 / q} \\
& \leq\left(x^{(p-1) / 2}\right)^{2 / q}=x^{(p-1) / q},
\end{aligned}
$$

or, in terms of the height,

$$
h(\alpha) \leq \frac{p-1}{2 q} \log x .
$$

This implies, with the notation of Theorem 3.1, that

$$
12.85 \times 2 \pi+\frac{p-1}{2 q} \log x<\frac{p}{2 q} \log x ;
$$

indeed, (2.1) says that $|x|>p^{q^{2} / p}$, so that

$$
\frac{1}{2 q} \log |x|>12.85 \times 2 \pi
$$

because of (2.3). Thus we can take

$$
t=\frac{p}{2 q} \log x
$$

(which implies $t>\frac{1}{2} q \log p$ ), and then we have

$$
H \leq \max \{17, \log q+3.46\} .
$$

(Proof: We have $0<k<q$ and $t>\frac{1}{2} q \log p$, so

$$
\begin{aligned}
D \log \left(\frac{b_{1}}{2 a}+\frac{b_{2}}{25.7 \pi}\right) & +4.6 D+3.25 \\
& <\log \left(\frac{q}{25.7 \pi}\right)+\frac{25.7 \pi}{2 a}+7.85 \\
& <3.459
\end{aligned}
$$

which proves that $H \leq \max \{17, \log q+3.46\}$.) Comparing the lower bound of $\log |\Lambda|$ with its upper bound, after some easy simplifications, we get

$$
q \leq 4.51 p H^{2}=4.51 p(\max \{17, \log q+3.46\})^{2} .
$$

This upper bound, like (2.13) and (2.14), is derived here as a reference for possible further computations. Note that it is better than (2.13) for $p \geq 31$.

\section{ACKNOWLEDGEMENT}

We are very grateful to one of the referees for pointing out several minor mistakes and obscurities and for simplifying considerably the proof of the better bound in Section 2 (pages 263 and following).

\section{REFERENCES}

[Cassels 1960] J. W. S. Cassels, "On the equation $a^{x}-b^{y}=1$, II", Proc. Cambridge Society 56 (1960), 97-103. 
[Hyyrö 1964] S. Hyyrö, "Über das Catalansche Problem", Ann. Univ. Turku, Ser. AI 79 (1964), 10 pages.

[Inkeri 1964] K. Inkeri, "On Catalan's problem", Acta Arith. 9 (1964), 285-290.

[Inkeri 1990] K. Inkeri, "On Catalan's conjecture", $J$. Number Theory 34 (1990), 142-152.

[Ko 1965] Ko Chao, "On the diophantine equation $x^{2}=y^{n}+1, x y \neq 0$ ", Sci. Sinica 14 (1965), 457-460.

[Langevin 1977] M. Langevin, "Quelques applications de nouveaux résultats de van der Poorten", Sém. Delange-Pisot-Poitou (1977/78), Paris, Exp. 4, 7 pages.

[Laurent et al.] M. Laurent, M. Mignotte, and Y. Nesterenko, "Formes linéaires en deux logarithmes et déterminants d'interpolation", to appear in J. Number Theory.

[Mignotte 1992] M. Mignotte, "Sur l'équation de Catalan", C. R. Acad. Sci. Paris, Sér. I 314 (1992), 165-168.
[Mignotte 1993] M. Mignotte, "Un critère élémentaire pour l'équation de Catalan", C. R. Math. Rep. Acad. Sci. Canada 15 (1993), 199-200.

[Mignotte 1994] M. Mignotte, "Sur l'équation de Catalan (II)", Theoret. Comp. Sci. 123 (1994), 145149.

[Mignotte 1995] M. Mignotte, "A criterion on Catalan's equation", J. Number Theory 52 (1995), 280-283.

[Nagell 1920] "Des équations indéterminées $x^{2}+x+1=$ $y^{n}$ et $x^{2}+x+1=3 y^{n}$ ", Nordsk. Mat. Forenings, Skr. (1) 2 (1920), 14 pages.

[Ribenboim 1994] P. Ribenboim, Catalan's conjecture, Academic Press, Boston, 1994.

[Tijdeman 1976] R. Tijdeman, "On the equation of Catalan", Acta Arith. 29 (1976), 197-209.

[Schwarz 1995] W. Schwarz, "A note on Catalan's equation", Acta Arith. 72 (1995), 277-279.

[Washington 1982] L. C. Washington, Introduction to cyclotomic fields, Springer, New York, 1982.

Maurice Mignotte, Université Louis Pasteur, Centre de Calcul de l'Esplanade, 7, rue René Descartes, F-67084 Strasbourg, France (mignotte@math.u-strasbg.fr)

Yves Roy, Université Louis Pasteur, Centre de Calcul de l'Esplanade, 7, rue René Descartes, F-67084 Strasbourg, France (yr@dept-info.u-strasbg.fr)

Received December 27, 1994; accepted in revised form August 8, 1995 